OPEN ACCESS

Edited by:

Yiliang Chen,

Medical College of Wisconsin,

United States

Reviewed by:

Bishuang Cai

Icahn School of Medicine at Mount

Sinai, United States

Jue Zhang,

Versiti Blood Research Institute,

United States

${ }^{*}$ Correspondence:

Wanxing Zhou

zhouwanx2015@sina.com

Specialty section:

This article was submitted to

Lipids in Cardiovascular Disease,

a section of the journal

Frontiers in Cardiovascular Medicine

Received: 19 April 2021 Accepted: 07 September 2021

Published: 01 October 2021

Citation:

Liao Y, Zhu E and Zhou W (2021)

Ox-LDL Aggravates the Oxidative Stress and Inflammatory Responses of THP-1 Macrophages by Reducing the Inhibition Effect of miR-491-5p on

MMP-9.

Front. Cardiovasc. Med. 8:697236.

doi: $10.3389 /$ fcrm.2021.697236

\section{Ox-LDL Aggravates the Oxidative Stress and Inflammatory Responses of THP-1 Macrophages by Reducing the Inhibition Effect of miR-491-5p on MMP-9}

\author{
Yiling Liao, Enzheng Zhu and Wanxing Zhou*
}

Department of Internal Cardiology Medicine, The First Affiliated Hospital/School of Clinical Medicine of Guangdong Pharmaceutical University, Guangzhou, China

Background: Oxidized low-density lipoprotein (ox-LDL) can induce oxidative stress and inflammatory responses in macrophages to facilitate the genesis and development of atherosclerosis. However, the intermediate links remain unclear. MiR-491-5P can inhibit matrix metalloproteinase 9 (MMP-9); however, it remains unclear whether ox-LDL enhances MMP-9 expression and aggravates the oxidative stress and inflammatory responses under the mediating effect of miR-491-5P.

Method: THP-1 macrophages were divided into 10 groups: blank (control), model (ox-LDL), miR-491-5P high-expression (miR-491-5P mimic), miR-491-5P control (mimic-NC), MMP-9 high-expression (MMP-9-plasmid), MMP-9 control (plasmid-NC), miR-491-5P+plasmid-NC, miR-491-5P+ MMP-9-plasmid, MMP-9 gene silencing (MMP-9-siRNA), and gene silencing control (siRNA-NC). The cells were transfected for $48 \mathrm{~h}$ and then treated with $50 \mu \mathrm{g} / \mathrm{mL}$ of ox-LDL for $24 \mathrm{~h}$. MMP-9 mRNA and miR-491-5P expression levels in the cells were detected using reverse transcription-quantitative polymerase chain reaction, and the MMP-9 levels were detected with western blotting. The levels of oxidative stress factors (malondialdehyde [MDA]), reactive oxygen species (ROS), and antioxidant factors (superoxide dismutase [SOD]), and the expression levels of inflammatory factors (tumor necrosis factor [TNF- $\alpha$ ] and interleukin-1 $\beta$ and-6 [IL-1 $\beta$ and IL-6]) in the supernatant were detected with enzyme-linked immunosorbent assay.

Results: MDA, ROS, TNF- $\alpha, I L-1 \beta, I L-6$, and MMP-9 levels were increased, SOD activity was reduced, and miR-491-5P expression was downregulated in the ox-LDL group compared to the control group. In the miR-491-5P mimic group, the MDA, ROS, TNF- $\alpha, I L-1 \beta, I L-6$, MMP-9 mRNA and protein levels were downregulated, and SOD activity was enhanced compared to the ox-LDL group. MMP-9-plasmid elevated the MDA, ROS, TNF- $\alpha, I L-1 \beta, I L-6, M M P-9$ mRNA and protein levels, and downregulated SOD activity and miR-491-5P expression. Following transfection with MMP-9-siRNA, the MMP-9-plasmid outcomes were nullified, and the resulting trends were similar to the miR-491-5p simulation group. Oxidative stress and inflammatory responses were higher 


\begin{abstract}
in the miR-491-5P mimic+MMP-9-plasmid co-transfection group than in the miR-491-5P mimic group.
\end{abstract}

Conclusion: Ox-LDL aggravates the oxidative stress and inflammatory responses of THP-1 macrophages by reducing the inhibition effect of miR-491-5p on MMP-9.

Keywords: ox-LDL, miR-491-5P/MMP-9 axis, THP-1 macrophages, oxidative stress, inflammatory response

\section{INTRODUCTION}

Oxidative stress and the inflammatory response play critical roles in the development of atherosclerosis (AS) (1). Lowdensity lipoprotein (LDL) is the primary lipoprotein involved in cholesterol-induced AS. Oxidized LDL (ox-LDL) is extremely pro-inflammatory and has pro-oxidative stress effects (2, 3). Macrophages, derived from mononuclear cells (1), can phagocytize ox-LDL to form lipid-laden foam cells, induce the generation of reactive oxygen species (ROS), secrete proinflammatory factors, and cause inflammation of vascular walls and their subsequent repair, leading to the genesis and development of plaques $(4,5)$. Matrix metalloproteinase 9 (MMP-9) is a proteolytic enzyme that can degrade the extracellular matrix (ECM) in plaque fibrous caps, weakening them and causing plaque instability $(6,7)$. MMP-9 is present at high expression levels in unstable plaques (8). MMP-9 exerts a positive feedback effect on many pro-inflammatory factors (9) and can aggravate the inflammatory response (10, 11), leading to the development and instability of plaques (12). Ox-LDL can induce macrophages to secrete MMP9 (13). However, the complete bio-molecular mechanism is not comprehensively understood. MiR-491-5P is a micro-RNA (miRNA), a micromolecular non-coding RNA with a length of 20-22 nucleotides (14). It can inhibit the expression of target mRNA by binding to it, thus exerting a regulatory effect $(15,16)$. MiR-491-5P achieves targeted inhibition of MMP-9 expression (17). When the regulatory effect of miR-491-5P on MMP-9 is removed, MMP-9 expression is upregulated, thus increasing the incidence of atherosclerotic cerebral infarction (18). However, whether ox-LDL enhances MMP-9 expression and aggravates the oxidative stress and inflammatory responses under the mediating effect of miR-491-5P remains unclear. To address this gap in understanding, in vitro cell experiments were conducted and are described here.

\section{MATERIALS AND METHODS}

\section{Cell Culture and Grouping}

Human monocytes (THP-1) were purchased from the Cell Bank of the Chinese Academy of Sciences. The cells were cultured in RPMI-1640 culture medium (Gibco; Thermo Fisher Scientific, Inc.) supplemented with 10\% FBS (Gibco; Thermo Fisher Scientific, Inc.). The F5 cells were incubated in a complete medium containing $100 \mathrm{ng} / \mathrm{mL}$ phorbol-12-phorbol myristate acetate (Sigma-Aldrich; Merck KGaA) for $48 \mathrm{~h}$ and differentiated into THP-1 macrophages as the experimental cells.
The experimental cells were divided into 10 groups: control, model (ox-LDL), miR-491-5P high-expression (miR491-5P mimic), miR-491-5P no-load (mimic-NC), MMP-9 high-expression (MMP-9-plasmid), MMP-9 no-load (plasmidNC), miR-491-5P+plasmid-NC, miR-491-5P+ MMP-9-plasmid, MMP-9 gene silencing (MMP-9-siRNA), and gene silencing control (siRNA-NC). Six wells were used in each group.

\section{Cell Transfection}

The oligonucleotide sequences (Gene Pharma, Shanghai, China) of miR-491-5P mimic (miR-491-5P imported) and miR-4915P mimic-NC (miR-491-5P control), MMP-9 gene silencing (MMP-9-siRNA), gene silencing control (siRNA-NC), MMP9 high-expression (MMP-9-plasmid), and plasmid control (plasma-NC) sequences (Santa Cruz Biotechnology) were used to establish the corresponding transfection systems. In each group, lipofectamine ${ }^{\mathrm{TM}} 2000$ transfection reagent (Invitrogen Life Technologies, CA, USA) was used to transfect the THP-1 macrophages for $48 \mathrm{~h}$.

\section{Co-Incubation of Ox-LDL and Transfected Macrophages}

The differentiated THP-1 macrophages were incubated using $10,30,50$, or $70 \mu \mathrm{g} / \mathrm{mL}$ ox-LDL (Sigma, USA) in an incubator (temperature: $37^{\circ} \mathrm{C}$, saturated humidity: $5 \% \mathrm{CO}_{2}$ ) for $24 \mathrm{~h}$ to determine the appropriate ox-LDL concentration (19). The transfected THP-1 macrophages were then treated with $50 \mu \mathrm{g} / \mathrm{mL}$ of ox-LDL (Sigma) for $24 \mathrm{~h}$.

\section{Analysis of Cell Viability}

The cell counting kit-8 (CCK-8) assay was used to analyze cell viability (Abcam, USA). Briefly, THP-1 cells were plated in 96well plates and differentiated into macrophages. After exposure to $50 \mu \mathrm{g} / \mathrm{mL}$ ox-LDL and cell transfection, $10 \mu \mathrm{L}$ of CCK- 8 working solution was added to each well, and incubation was continued for $4 \mathrm{~h}$ at $37^{\circ} \mathrm{C}$. The plates were detected at $450 \mathrm{~nm}$ using a spectrophotometer (Thermo Fisher Scientific).

\section{Verification of THP-1 Macrophage Foaming Oil Red O Staining}

THP-1 macrophages were incubated with or without ox-LDL and transfected with miR-491-5P mimic, MMP-9-plasmid, MMP-9siRNA, or miR-491-5P mimic+MMP-9-plasmid for $48 \mathrm{~h}$ before exposure to $50 \mu \mathrm{g} / \mathrm{mL}$ ox-LDL. After washing three times with phosphate-buffered saline (PBS), the cells were fixed with $4 \%$ paraformaldehyde for $25 \mathrm{~min}$. Oil Red $\mathrm{O}$ solution (for cultured cells) was added and the cells were incubated for $30 \mathrm{~min}$ at $37^{\circ} \mathrm{C}$ and counterstained with Mayer Hematoxylin solution, according 
to the manufacturer's protocol (G1262 Solarbio Beijing, China). After extensive washing with PBS, the cells were immediately photographed using a microscope (Olympus). After removing the staining solution, the dye retained in the cells was eluted into isopropanol and the IOD value of the Oil Red O staining was detected at $540 \mathrm{~nm}$ using a spectrophotometer (Thermo Fisher Scientific).

\section{Lipid Accumulation Assay}

Lipid drop (LD) accumulation was evaluated using BODIPY 493/503 (10 $\mu \mathrm{g} / \mathrm{mL}$; Thermo Fisher, D2148) staining. The nuclei were stained with 4',6-diamidino-2-phenylindole (DAPI) staining solution. The average LD intensity from BODIPY $493 / 503$ staining was quantified using Image J software (National Institutes of Health).

\section{Reverse Transcription-Quantitative Polymerase Chain Reaction (RT-qPCR)}

Total RNA was extracted from the cells using TRIzol ${ }^{\circledR}$ reagent (Invitrogen; Thermo Fisher Scientific, Inc.). Hi Script TM II Q RT Super Mix (Vazyme Biotech Co., Ltd.) was used for the reverse transcription of total RNA into cDNA. Subsequently, a SYBR Green PCR kit (Vazyme Biotech Co., Ltd.) was used for qPCR. Quantification was undertaken using the 2- $\Delta \Delta$ Cq method (20), and the internal references were GAPDH (for mRNA) and U6 (for miRNA). The assays were repeated three times for each sample and the mean value was used. The PCR primer sequences were as follows:

$$
\begin{aligned}
& \text { MiR-491-5P forward 5'-GGAGT GGGG AACCCTTCC-3'; } \\
& \text { reverse 5' -GTGGGGGAGGGGATTC- } 3^{\prime}
\end{aligned}
$$

U6 forward 5'-GCTTC GGAGCACATACT AAAA T-3'; reverse 5'-CGCTTCACGAATTT GCGTGTCAT-3'

MMP-9 forward 5' -AGACCTGCGAGGAGATTCCAA3'; reverse 5'-CGGAGGAGTCGAGT-3';

GAPDH forward 5' -CTTGGGGGTA GGAAGGACTC-3' reverse $5^{\prime}$-GTA GAGAGGAGATGATGTTCT-3'.

\section{Western Blotting Analysis}

The cells were pyrolyzed using RIPA pyrolytic buffer solution (Beyotime Institute of Biotechnology, Shanghai, China), and the total protein was extracted. A BCA Protein Assay kit (Beyotime Institute of Biotechnology, Shanghai, China) was used to quantify the total protein. The equivalent amount of protein was treated for $40 \mathrm{~min}$ with $12 \%$ SDS-PAGE Gel SuperQuick Preparation Kit for electrophoretic separation. The separated protein was transferred to polyvinylidene difluoride membranes (Millipore Corporation) and sealed using 5\% skim milk to prevent nonspecific binding. Next, the membranes were incubated with the indicated primary antibodies, including MMP-9 (1:1000, Abcam), and left overnight at $4^{\circ} \mathrm{C}$. The primary antibodies were rinsed, and the membrane was incubated with secondary antibodies for $2 \mathrm{~h}$; anti-GAPDH antibody (1:1000, Abcam) was used as the internal reference. After rinsing, the protein bands were assessed using an enhanced chemiluminescence reagent (WBKLS0100, Millipore, USA).

\section{Detection of Oxidative Stress-Related Factors}

A superoxide dismutase (SOD) assay kit (Cayman Chemical Company) was used to determine SOD activity, as per the manufacturer's protocol. Malondialdehyde (MDA) activity was measured using an MDA assay kit (Cayman Chemical Company), according to the manufacturer's protocol. ROS levels were analyzed using a specific kit.

\section{Enzyme-Linked Immunosorbent Assay (ELISA)}

The concentrations of inflammatory cytokines, including tumor necrosis factor $\alpha$ (TNF- $\alpha$ ), interleukin-1 $\beta$ (IL-1 $\beta$ ), and IL-6, were detected using ELISA kits ( $\&$ \& D, USA). Specific ELISA kits were used to detect the related markers, according to the manufacturer's protocols.

\section{Statistical Analysis}

Statistical analysis was performed using SPSS 26.0, followed by graphical analysis using GraphPad Prism 8.0 software (GraphPad Software Company). The normal distribution test and homogeneity test of variance were undertaken initially, and the normally distributed data were expressed as the mean \pm standard deviation (SD). The least significant difference test was used to compare every two groups for homogeneity of variance. One-way analysis of variance was undertaken for multigroup comparisons; non-parametric tests were used in the case of heterogeneity of variance. $P<0.05$ was interpreted as a statistically significant difference.

\section{RESULTS}

\section{Ox-LDL Downregulated miR-491-5P in THP-1 Macrophages}

To investigate the effect of ox-LDL on THP-1 macrophages, THP-1 macrophages were treated with ox-LDL at different concentrations $(0,10,30,50$, and $70 \mu \mathrm{g} / \mathrm{mL})$ for $24 \mathrm{~h}$. OxLDL significantly reduced THP-1 macrophage activity and mir491-5p expression and upregulated MMP-9 levels in a dosedependent manner (Figures 1A-C). There was no significant difference between the $50 \mu \mathrm{g} / \mathrm{mL}$ and $70 \mu \mathrm{g} / \mathrm{mL}$ ox-LDL treatments; therefore, the $50 \mu \mathrm{g} / \mathrm{mL}$ concentration was selected for subsequent experiments.

\section{Ox-LDL Induced Oxidative Stress and Inflammatory Response of THP-1 Macrophages by Regulating mir-491-5p and MMP-9}

Subsequently, the expression levels of MMP9 and miR-491$5 \mathrm{p}$ in ox-LDL-induced THP-1 macrophages oxidative stress and inflammatory response were detected. The ox-LDL group had significantly higher ROS and MDA levels and lower SOD activity than the control group (Figures 2A-C). ELISA results showed that the ox-LDL group increased the secretory levels of TNF- $\alpha$, IL-1 $\beta$, and IL-6 (Figure 2D). RT-qPCR/western blotting 

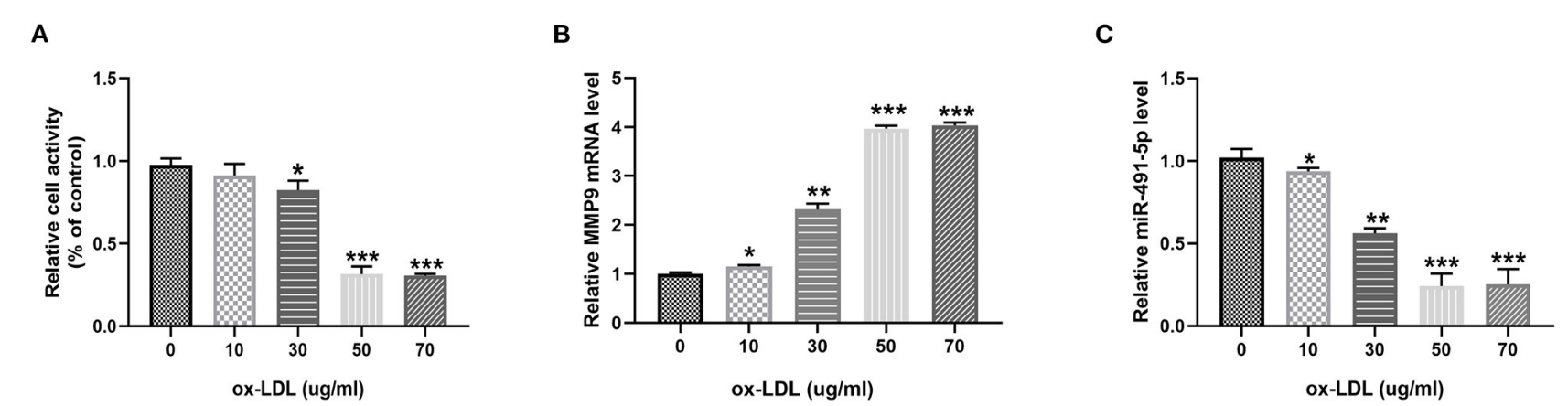

FIGURE 1 | Ox-LDL downregulated miR-491-5P and upregulated MMP-9 in THP-1 macrophages. THP-1 macrophages were induced by different concentrations (0, 10, 30, 50, and $70 \mu \mathrm{g} / \mathrm{mL}$ ) of ox-LDL. (A) Cell viability was determined using CCK-8. (B,C) qRT-PCR analysis measured MMP-9 level and mir-491-5p level in THP-1 macrophages stimulated with different doses of ox-LDL. Data are presented as the mean $\pm \mathrm{SD}, n=6$. ${ }^{\star} P<0.05$, ${ }^{\star \star} P<0.01$, and ${ }^{\star \star \star} P<0.001$.

analyses showed that ox-LDL induced downregulation of miR491-5p expression (Figure 2E) and upregulation of MMP-9 protein (Figure 2F) and mRNA (Figure 2G) expression in THP-1 macrophages. These results indicated that mir-491-5P and MMP9 might mediate oxidative stress and inflammatory response induced by ox-LDL.

\section{MMP-9 Overexpression and Knockdown Promoted and Inhibited Ox-LDL-Induced Oxidative Stress and Inflammatory Response in THP-1 Macrophages, Respectively}

Next, protein and mRNA expression levels of MMP-9 in THP1 macrophages were upregulated via gene introduction in the MMP-9-plasmid group and downregulated in the MMP9-siRNA group (Figures 3A-D). RT-qPCR and western blotting analyses demonstrated the ox-LDL treatment downregulated mir-491$5 \mathrm{p}$ (Figure 3E) and upregulated MMP-9 protein (Figure 3F) and mRNA (Figure 3G) expression levels. These changes were intensified by MMP-9-plasmid and suppressed by MMP-9siRNA (Figures 3E-G). The MMP-9-plasmid group had higher ROS (Figure 3H) and MDA (Figure 3I) release and lower SOD activity (Figure 3J) than the ox-LDL group; however, the MMP9siRNA group had significantly lower ROS and MDA release and higher SOD activity than the ox-LDL group (Figures $\mathbf{3 H - J}$ ). In addition, the ox-LDL treatment markedly increased the secretory levels of TNF- $\alpha$, IL-1 $\beta$, and IL-6 (Figure 3K); however, these changes were aggravated by MMP-9-plasmid and inhibited by MMP9-siRNA. These results hinted that MMP-9 mediated oxLDL-induced oxidative stress and inflammatory response in THP-1 macrophages.

\section{MMP-9 Overexpression Promoted Ox-LDL-Induced THP-1 Macrophage Foaming}

Meanwhile, to further investigate the effect of MMP-9 on oxLDL-induced cell foaming, we evaluated the intracellular lipid accumulation of both MMP-9 overexpression or knockdown in THP-1 macrophages by oil red staining and fluorescence microscopy. The results showed that cytoplasmic lipid drops staining and quantitative analysis were significantly increased in the ox-LDL group, indicating THP-1 macrophage foaming (Figures 4A,B). The MMP-9-plasmid group had significantly aggravated THP-1 macrophage foaming (Figure 4A) and lipid uptake (Figure 4B) compared to the ox-LDL group, whereas MMP-9-siRNA significantly reduced ox-LDL-induced macrophage foaming and lipid uptake (Figures 4A,B). These data identified that MMP-9 not only worsened ox-LDL-induced THP-1 macrophage oxidative stress and inflammatory response but also caused cell foaming.

\section{MiR-491-5p Inhibited Ox-LDL-Induced THP-1 Macrophages Inflammation and Oxidative Stress by Downregulating MMP-9 Expression}

To further demonstrate whether the effects of mir-491-5p on inflammatory cytokines and oxidative stress induced by ox-LDL by regulating MMP-9 in THP-1 macrophage cells, experiments were carried out through the transduction of mir491-5p mimic or mir-491-5p mimic+MMP-9 plasmid into THP-1 macrophage cells before treatment with $50 \mu \mathrm{g} / \mathrm{mL}$ oxLDL. After transduction of miR-491-5p into macrophages in the miR-491-5p mimic group, miR-491-5p expression was upregulated (Figure 5A), and mRNA (Figure 5B) and protein (Figure 5C) expression levels of MMP-9 in THP-1 macrophages were significantly downregulated. These changes were reversed after co-transfection with the MMP9-plasmid in the miR491-5p mimic+ MMP-9-plasmid group (Figures 5B,C). To investigate the role of mir-491-5P in ox-LDL-mediated damage, THP-1 macrophages transduced with mir-491-5p mimic or mimic-NC were stimulated with $50 \mu \mathrm{g} / \mathrm{mL}$ ox-LDL. CCK-8 analysis showed that high mir-491-5p expression increased the viability of THP-1 macrophages compared to the oxLDL treatment alone (Figure 5D). MMP-9 mRNA (Figure 5E), protein (Figure 5F), ROS, MDA (Figures 5G,H), IL-6, IL-1 $\beta$, and TNF- $\alpha$ (Figure 5J) expression levels were downregulated, 


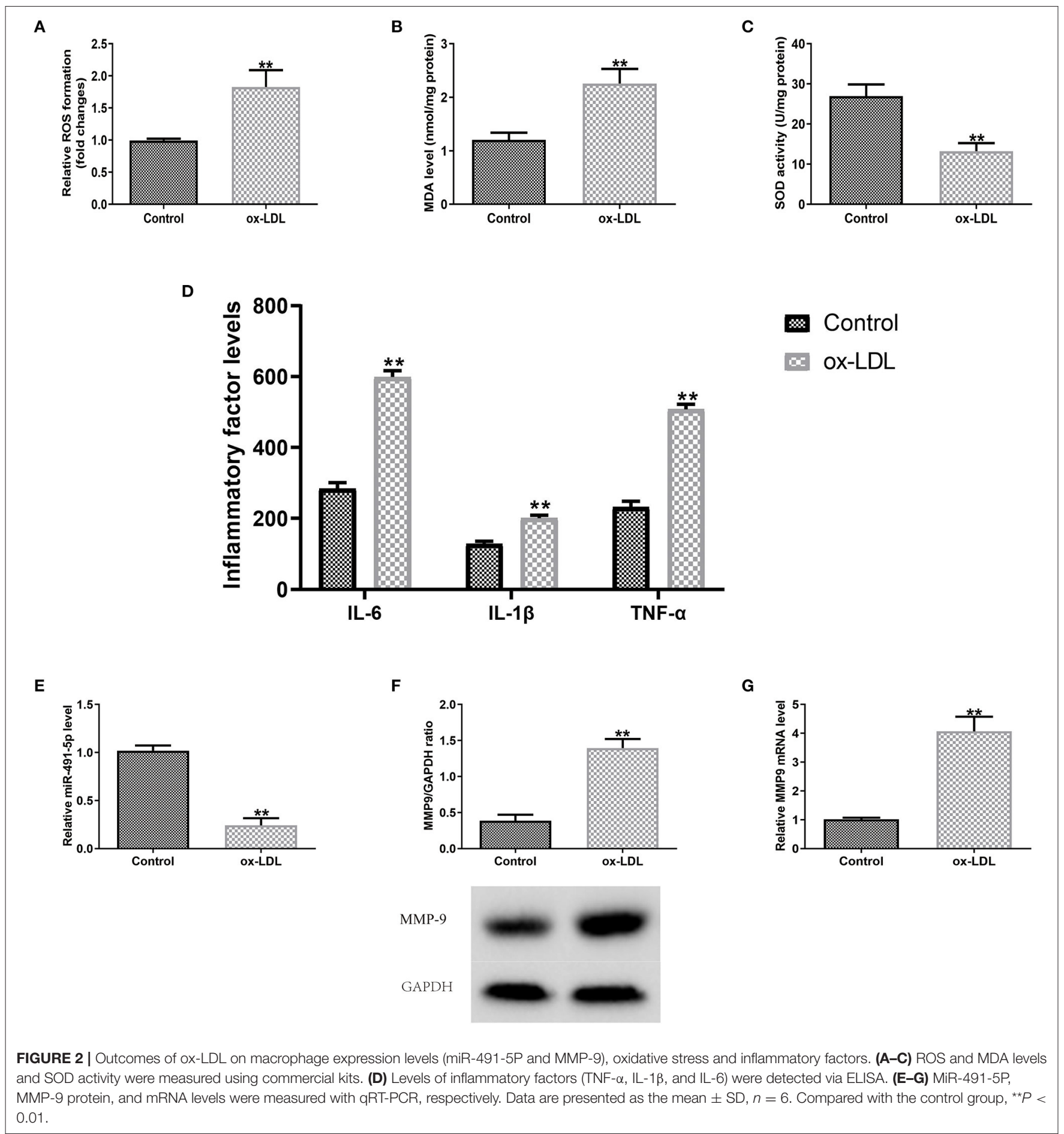

and SOD activity was improved (Figure 5I) in the miR-4915P mimic group compared to the ox-LDL group. In contrast, these observational indicators were reversed in the miR-4915P mimic+MMP-9-plasmid group (Figures 5E-J). These results suggested that mir-491-5p inhibited ox-LDL-induced oxidative stress and inflammatory cytokines in THP-1 macrophages by inhibiting MMP-9 expression.

\section{Mir-491-5p Attenuated Ox-LDL-Induced THP-1 Macrophages Foaming}

Similarly, we observed the effect of mir-491-5p on ox-LDLinduced THP-1 macrophage foaming by oil red staining and fluorescence microscopy. Oil Red O and BODIPY 493/503 staining showed that ox-LDL induced the THP-1 macrophage foaming and increased content lipid droplets contentment 
A

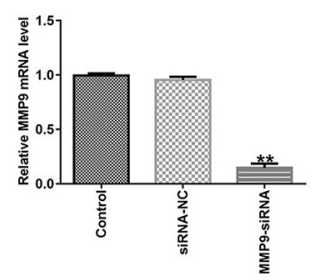

C

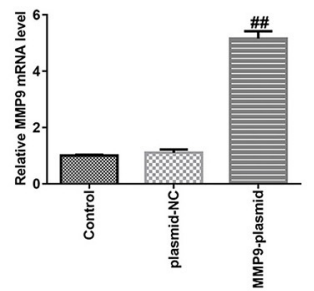

E

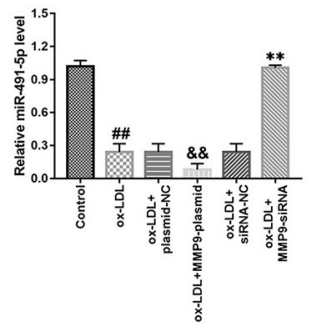

H

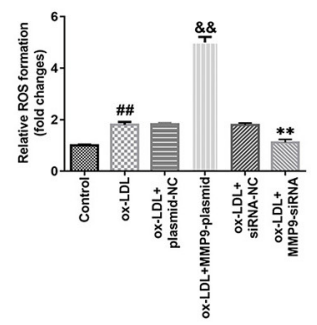

K

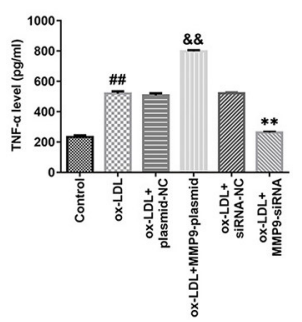

B

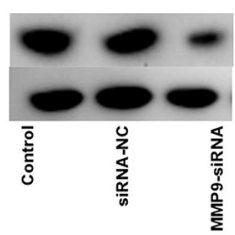

MMP-9

GAPDH

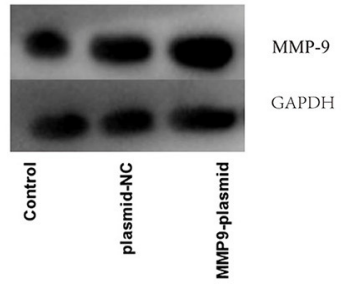

F

MMP-9

GAPDH

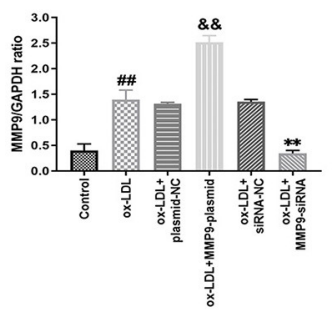

I
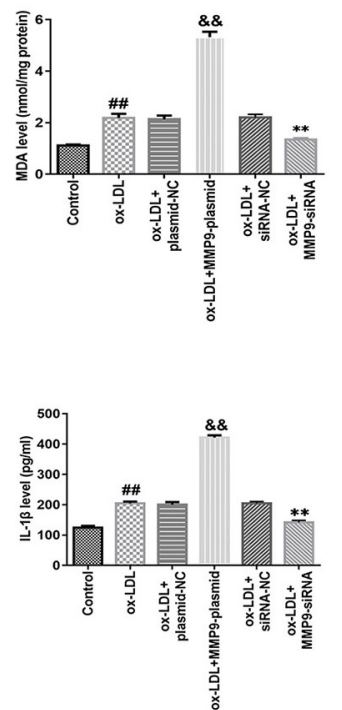
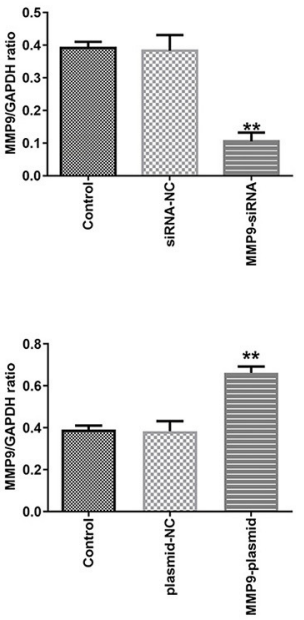

G

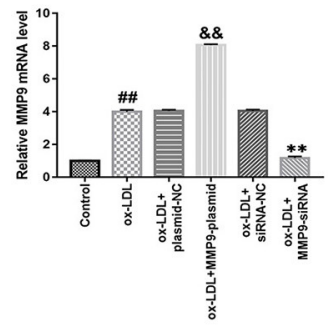

J
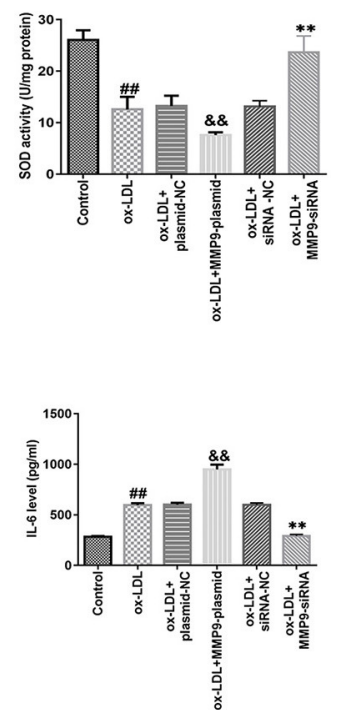

FIGURE 3 | Effect of MMP-9 on the oxidative stress and inflammatory reactions in ox-LDL-induced THP-1 macrophages. (A-D) THP-1 macrophages were introduced with MMP-9-siRNA and MMP-9-plasmid. Transfection efficiency of MMP-9-plasmids and MMP-9-siRNA were measured with qRT-PCR, whereas MMP-9 protein was measured with western blotting. (E-K) Transfected THP-1 macrophages were stimulated with $50 \mu \mathrm{g} / \mathrm{mL}$ ox-LDL. (E) MiR-491-5P expression of transfected THP-1 macrophages was determined with qRT-PCR. (F,G) MMP-9 protein and mRNA expression of transfected THP-1 macrophages was determined by western blotting and GRT-PCR, respectively. (H-J) ROS and MDA levels and SOD activity were measured using commercial kits. (K) Levels of inflammatory factors (TNF- $\alpha, I L-1 \beta$, and IL-6) were detected using ELISA. Data are presented as the mean \pm SD, $n=6$. Compared with the control group, \#\#P<0.01; compared with the plasmid-NC group, ${ }^{\& \&} P<0.01$; compared with the si-RNA-NC group, ${ }^{\star \star} P<0.01$. 

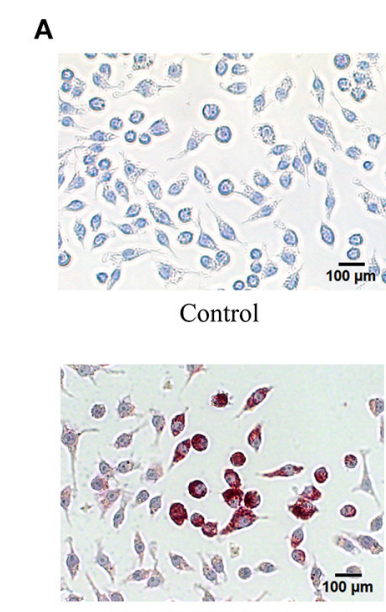

ox-LDL+MMP-9-plasmid

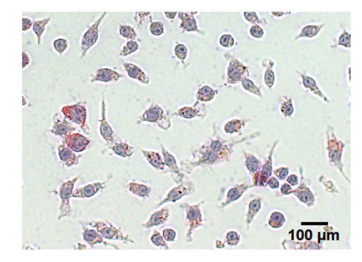

ox-LDL

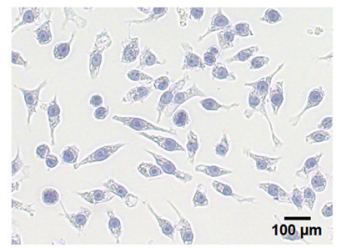

ox-LDL+MMP-9-si-RNA
B

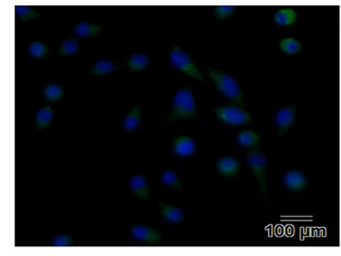

Control

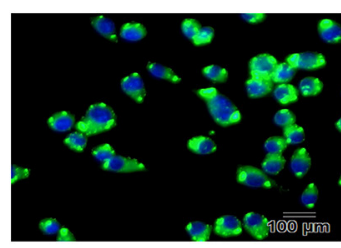

ox-LDL+MMP-9-plasmid

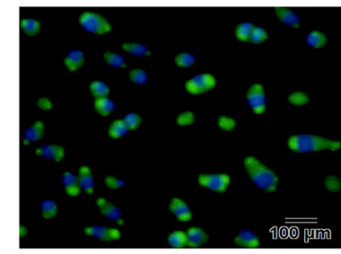

ox-LDL

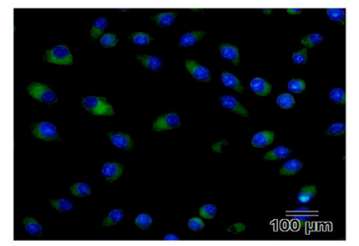

ox-LDL+MMP-9-si-RNA
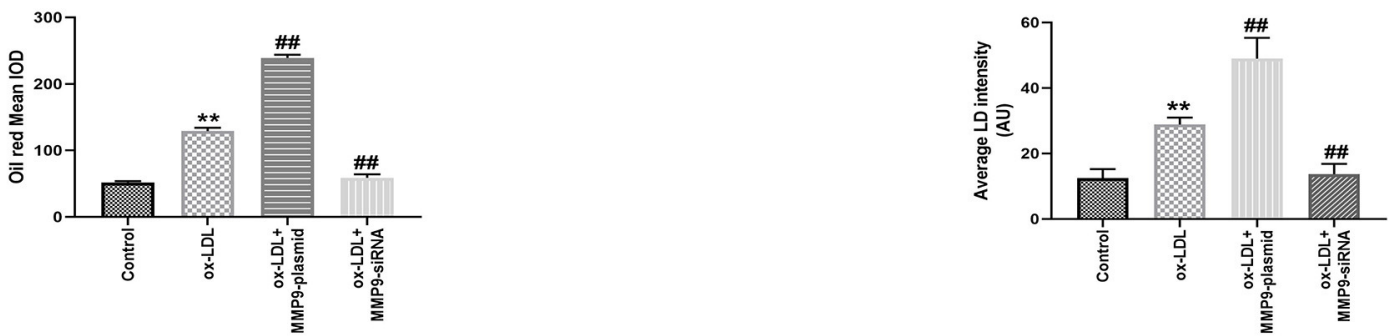

FIGURE 4 | Oil Red O and BODIPY 493/503 staining after MMP-9-plasmid and MMP-9-siRNA transfections. (A) THP-1 macrophage cells were transfected before incubation with ox-LDL for $24 \mathrm{~h}$ and stained with Oil Red O ( $\times 200,100 \mu \mathrm{m})$, the intracellular lipid droplets of the mean optical density value (mean IOD) were used to reflect the degree of cellular foam. (B) Lipid droplets were labeled with green fluorescence and the nuclei were labeled with DAPI (blue) ( $\times 200,200 \mu \mathrm{m})$, average LD intensity was used to quantify lipid uptake. Data are presented as the mean $\pm \mathrm{SD}, n=6$. Compared with the control group, ${ }^{\star \star} P<0.01$; compared with the ox-LDL group, $\# \# p<0.01$.

in cells (Figures 6A,B). The mir-491-5P mimic group had significantly lower THP-1 macrophage foaming (Figure 6A) and lipid uptake (Figure 6B) than the ox-LDL group, whereas the mir-491-5P mimic+MMP-9-plasmid significantly reversed these phenomena (Figures 6A,B). These results suggested that miR491-5p inhibited ox-LDL-induced THP-1 macrophage foaming by inhibiting MMP-9 expression.

\section{DISCUSSION}

LDL is the primary lipoprotein inducing AS, and ox-LDL cannot be easily recognized and scavenged by the relevant receptors (3). The macrophages differentiated by monocytes can phagocytize ox-LDL to form lipid-laden foam cells, thus becoming a component of plaques $(4,5)$. Macrophage foaming and increased intracellular lipid content can be used as markers of the severity of arteriosclerosis caused by lipids. Macrophage foaming and lipid accumulation were visual after Oil Red $\mathrm{O}$ and BODIPY 493/503 staining, and the intracellular lipid droplets were quantitatively assessed using the mean optical density value (mean IOD) and average LD intensity, respectively. OxLDL can stimulate macrophages to release oxygen free radicals and inflammatory factors, resulting in local tissue necrosis and triggering and facilitating the formation of plaques (21). ROS and MDA levels reflect the lipid peroxidation degree, and SOD is an antioxidant factor (2). Upregulated expression of TNF- $\alpha$, IL-1 $\beta$, and IL-6 infer aggravation of the inflammatory response (12). Further, ox-LDL can stimulate macrophages to release MMP-9 and elevate MMP-9 expression level in plaques $(22,23)$. In the present study, after the macrophages were co-incubated with oxLDL, the MMP-9, ROS, MDA, TNF- $\alpha$, IL- $1 \beta$, and IL- 6 expression levels were upregulated in the model group. Simultaneously, SOD activity was reduced, and foam cell formation and lipid accumulation were increased, validating the expected effect of oxLDL on the macrophages, consistent with previous studies (19, 24). In contrast, the miR-491-5P expression level was lowered. Further investigations illustrated that the effects of ox-LDL on macrophages were lessened in the ox-LDL + miR-491-5P mimic group after the miR-491-5P expression level was elevated via gene introduction. This phenomenon was nullified in the ox-LDL + miR-491-5Pmimic+MMP-9plasmid group with high miR491-5P and MMP-9 expression levels. However, the MMP-9 expression level increased in the MMP-9-plasmid group, and the corresponding oxidative stress and inflammatory responses and the degree of cell foaming were enhanced. Those in the MMP9-siRNA group with MMP-9 silencing showed the opposite 
A
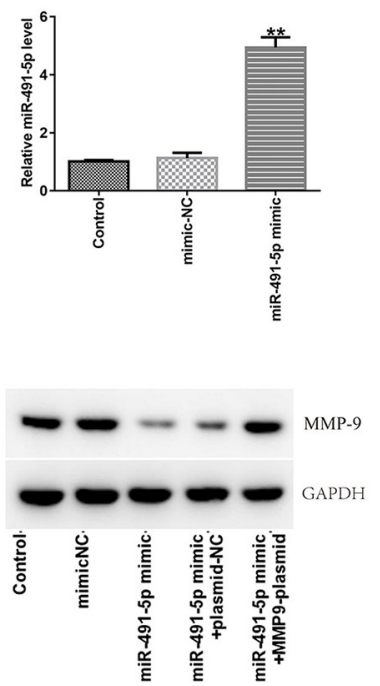

E

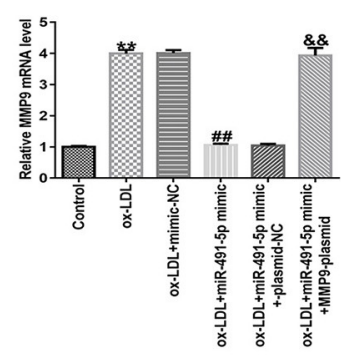

G
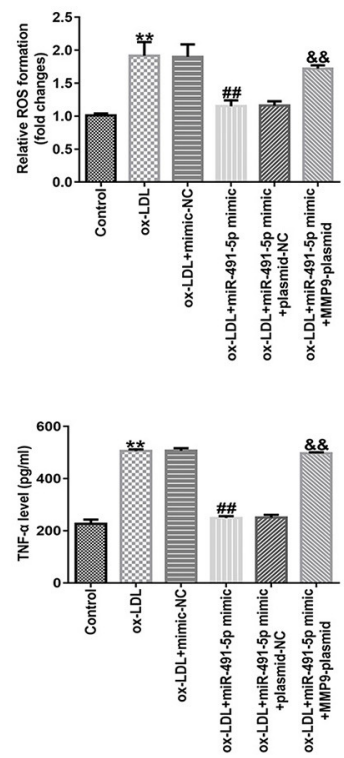

B
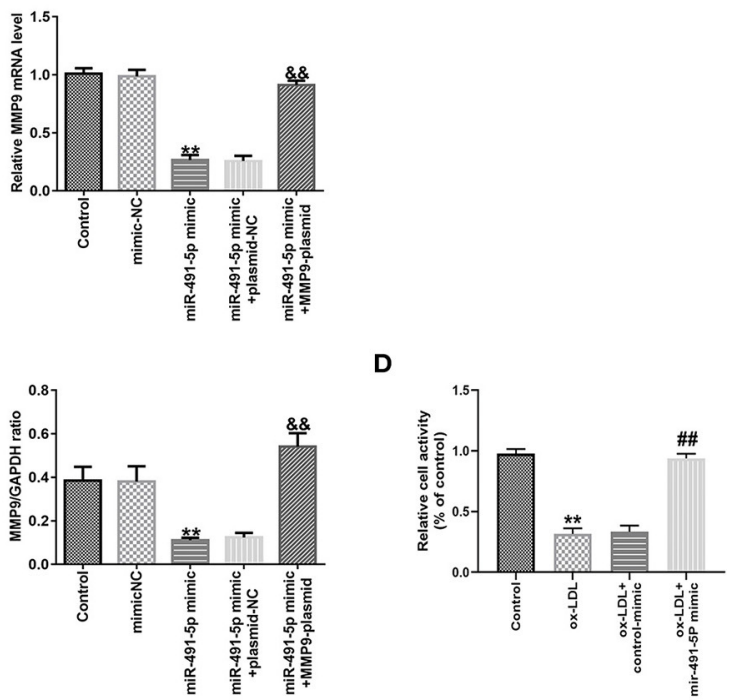

F
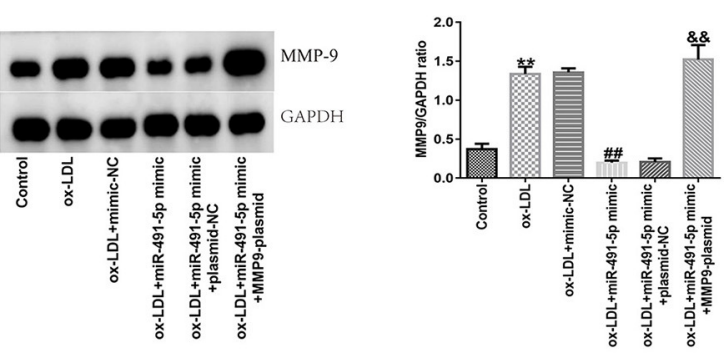

H

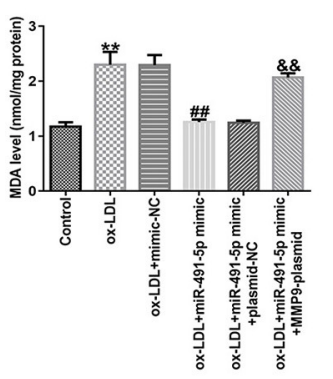

I
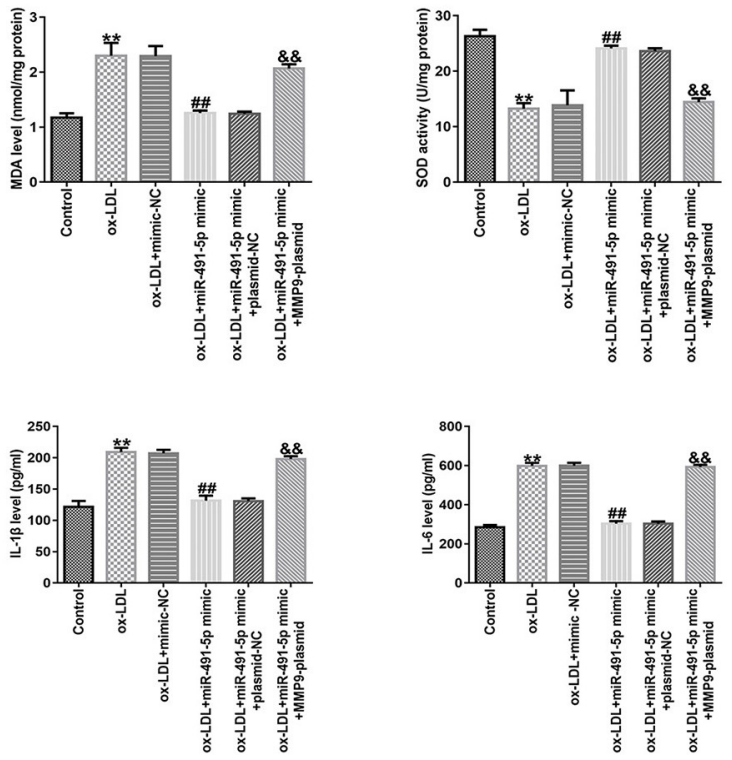

FIGURE 5 | Effect of miR-491-5p and MMP-9 on ox-LDL-induced THP-1 macrophages. (A) The transfection efficiency of miR-491-5P mimic was determined using qRT-PCR. (B,C) MMP-9 mRNA and protein levels were detected in THP-1 macrophages transduced with miR-491-5P and miR-491-5P mimic+MMP-9-plasmid. (D-J) Transfected THP-1 macrophages were stimulated with $50 \mu \mathrm{g} / \mathrm{mL}$ ox-LDL. (D) Cell activity after mir-491-5P mimic transfection was assessed using CCK-8. (E,F) MMP-9 mRNA and protein were detected in ox-LDL-induced THP-1 macrophages transduced with mir-491-5p mimic and mir-491-5p mimic +MMP-9-plasmid. (G-I) ROS and MDA levels and SOD activity were examined using commercial kits. (J) Levels of inflammatory factors (TNF- $\alpha$, IL-1 $\beta$, and IL-6) were measured with ELISA. Data are presented as the mean $\pm \mathrm{SD}, n=6$. Compared with the control group, ${ }^{\star \star} P<0.01$; compared with the ox-LDL+mimic-NC group, $\# \# p<0.01$; compared with the ox-LDL + miR-491-5Pmimic+plasmid-NC group, \&\& $P<0.01$ 


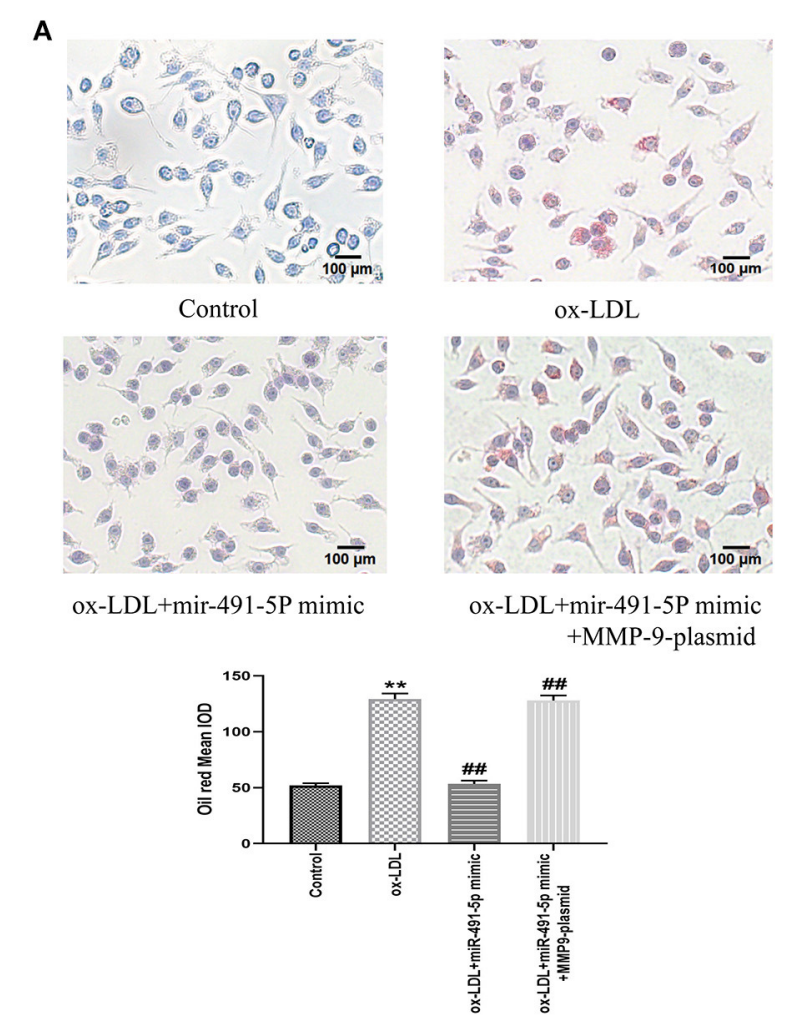

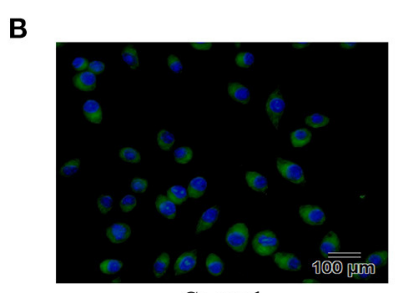

Control

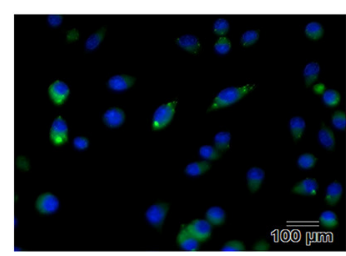

ox-LDL+mir-491-5P mimic

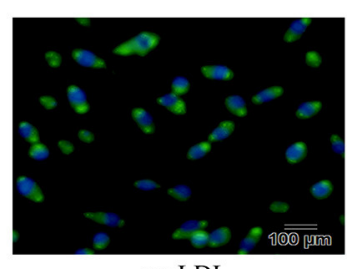

ox-LDL

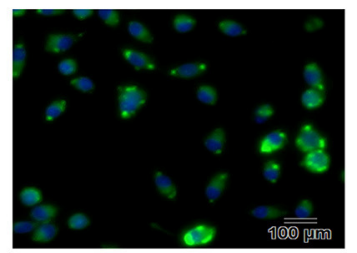

ox-LDL+mir-491-5P mimic +MMP-9-plasmid

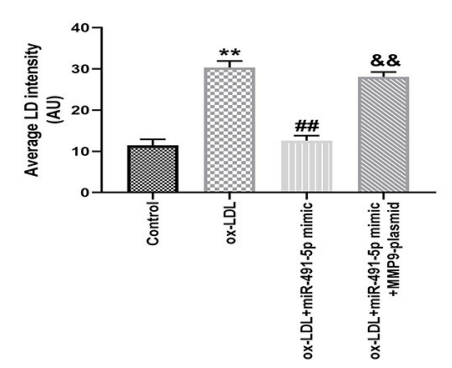

FIGURE 6 | Oil Red O and BODIPY 493/503 staining after mir-491-5P mimic and mir-491-5P mimic +MMP-9-plasmid transfections. (A) THP-1 macrophage cells were transfected before incubation with ox-LDL and staining with Oil Red $\mathrm{O}(\times 200,100 \mu \mathrm{m})$, the intracellular lipid droplets of the mean optical density value (mean IOD) were used to reflect the degree of cellular foam. (B) Lipid droplets were labeled with green fluorescence and the nuclei were labeled with DAPI (blue) $(\times 200$, $100 \mu \mathrm{m})$, average LD intensity was used to quantify the lipid uptake. Data are presented as the mean $\pm \mathrm{SD}, \mathrm{n}=6$. Compared with the control group, ${ }^{\star \star} P<0.01$; compared with the ox-LDL group, \#\# $p<0.01$; compared with the ox-LDL+mir-491-5pmimic group, \&\&p < 0.01 .

trend, indicating that ox-LDL might aggravate the oxidative stress and inflammatory responses of THP-1 macrophages via the repression of the miR-491-5P/MMP-9 axis.

Recently, extensive research attention has focused on the biological functions of miRNA. MiR-491-5P, a type of miRNA, can regulate the expression of the MMP-9 gene by binding to the $3^{\prime}$ UTR non-coding site of MMP-9 $(25,26)$. Recent in vitro studies have shown that miR-491-5P can inhibit the growth and migration of vascular smooth muscle cells through the targeted inhibition of MMP-9 expression (17). Furthermore, clinical studies have indicated that the binding site for miR-491-5P and MMP-9 undergoes mutation due to the gene polymorphism of MMP-9; therefore, miR-491-5P fails to bind to MMP-9, the in vivo MMP-9 expression level of the patient is increased, and the risk of atherosclerotic cerebral infarction is amplified (18). According to the present study, miR-491-5P repressed the oxLDL-induced oxidative stress and inflammatory responses of THP-1 macrophages through the targeted inhibition of MMP-9, and cell foaming and lipid uptake were significantly decreased, further demonstrating the role of the miR-491-5P/MMP-9 pathway in AS attack.

However, the upstream promoter of miR-491-5P must be further clarified. Transcription factors, epigenetic modification, and the conversion of pri-miR to miRNA might play essential roles in regulating miRNA expression. Previous tumor cell studies found that the Foxil protein, belonging to the forkhead gene family, is a potential upstream regulator of miR-491-5p, and can bind to miR-491-5p binding sites to regulate the expression of miR-491-5p (27). Another study found that circ0001361, a circular RNA member, can specifically inhibit miR-491-5P (28) and upregulate MMP-9 to promote cell proliferation and migration. Although the existing literature indicates that the Foxi1 protein and circ0001361 are upstream of miR-491-5p, it is unclear whether the Foxil protein or CIRC0001361 play a role in mediating the ox-LDL-induced oxidative stress and inflammatory responses of THP-1 macrophages. If so, it remains unclear what their upstream factors are. The pathway between ox-LDL and miR-491-5p appears to be complex and requires further exploration in future studies.

As a proteolytic enzyme, MMP-9 degrades the fibers in plaque ECM. The plaques become soft, fiber caps become thin, and the plaques become unstable, increasing the probability of clinical cardiovascular events (7). Another study has shown that MMP-9 exerts a "positive feedback" regulatory effect on inflammatory factors, and its high expression can aggravate the inflammatory response (9). IL- $1 \beta$ and IL- 8 are essential 
inflammatory cytokines; MMP-9 facilitates the transformation of the inactive precursor IL- $1 \beta$ into active IL- $1 \beta$ by activating the IL$1 \beta$ invertase (10) and pyrolyzes IL- 8 to enhance its chemotactic activity and further aggravate the inflammatory response (11). In inflammatory diseases, MMP-9 gene silencing can also restrict ROS accumulation (29), obstruct MMP-9 activation, repress the redox reaction, and relieve neuroinflammation (30). The present study showed that high MMP-9 expression further enhanced the oxidative stress and inflammatory responses to ox-LDL. MMP-9 silencing led to the opposite outcome, further verifying the oxidative stress-promoting and pro-inflammatory effects of MMP-9. Moreover, the gene introduction downregulated the miR-491-5P expression level under high MMP-9 expression. In the MMP-9 gene silencing condition, the miR-491-5P level was upregulated, suggesting that MMP-9 might exert negative feedback on miR-491-5P. Nevertheless, the mechanism remains unclear.

Several limitations need to be considered in the present study. The in vitro experiment confirmed that miR-491-5P/MMP-9 mediated a possible induction mechanism of ox-LDL in AS. However, as the formation of arterial plaques could not be directly observed, this requires further verification through follow-up in vivo and pathophysiological studies of plaque

\section{REFERENCES}

1. Tabas I, Lichtman AH. Monocyte-macrophages and T cells in atherosclerosis. Immunity. (2017) 47:621-34. doi: 10.1016/j.immuni.2017.09.008

2. Zhang Q, Ai Y, Dong H, Wang J, Xu L. Circulating oxidized low-density lipoprotein is a strong risk factor for the early stage of coronary heart disease. IUBMB Life. (2019) 71:277-82. doi: 10.1002/iub.1966

3. Tsimikas S, Witztum JL. Measuring circulating oxidized low-density lipoprotein to evaluate coronary risk. Circulation. (2001) 103:19302. doi: 10.1161/01.CIR.103.15.1930

4. Gui T, Shimokado A, Sun Y, Akasaka T, Muragaki Y. Diverse roles of macrophages in atherosclerosis: from inflammatory biology to biomarker discovery. Mediators Inflamm. (2012) 2012:693083. doi: 10.1155/2012/693083

5. Kockx MM, De Meyer GR, Bortier H, de Meyere N, Muhring J, Bakker A, et al. Luminal foam cell accumulation is associated with smooth muscle cell death in the intimal thickening of human saphenous vein grafts. Circulation. (1996) 94:1255-62. doi: 10.1161/01.CIR.94.6.1255

6. Ding S, Zhang M, Zhao Y, Chen W, Yao G, Zhang C, et al. The role of carotid plaque vulnerability and inflammation in the pathogenesis of acute ischemic stroke. Am J Med Sci. (2008) 336:27-31. doi: 10.1097/MAJ.0b013e3181 $5 \mathrm{~b} 60 \mathrm{a} 1$

7. Brown BA, Williams H, George SJ. Evidence for the involvement of matrixdegrading metalloproteinases (MMPs) in atherosclerosis. Prog Mol Biol Transl Sci. (2017) 147:197-237. doi: 10.1016/bs.pmbts.2017.01.004

8. Seifert R, Kuhlmann MT, Eligehausen S, Kiefer F, Hermann S, Schäfers M. Molecular imaging of MMP activity discriminates unstable from stable plaque phenotypes in shear-stress induced murine atherosclerosis. PLoS ONE. (2018) 13:e0204305. doi: 10.1371/journal.pone.0204305

9. Opdenakker G, Van den Steen PE, Dubois B, Nelissen I, Van Coillie E, Masure $\mathrm{S}$, et al. Gelatinase B functions as regulator and effector in leukocyte biology. $J$ Leukoc Biol. (2001) 69:851-9. doi: 10.1189/jlb.69.6.851

10. Schönbeck U, Mach F, Libby P. Generation of biologically active IL-1 beta by matrix metalloproteinases: a novel caspase-1-independent pathway of IL-1 beta processing. J Immunol. (1998) 161:3340-6.

11. Van den Steen PE, Proost P, Wuyts A, Van Damme J, Opdenakker G. Neutrophil gelatinase $B$ potentiates interleukin-8 tenfold by aminoterminal processing, whereas it degrades CTAP-III, PF-4, and formation. In addition, the specific mechanism underlying MMP-9 regulation by ox-LDL remains unclear.

In summary, ox-LDL was found to aggravate the oxidative stress and inflammatory reactions of THP-1 macrophages by downregulating the miR-491-5P expression level and reducing the inhibiting effect of miR-491-5P on MMP-9.

\section{DATA AVAILABILITY STATEMENT}

The original contributions presented in the study are included in the article/supplementary material, further inquiries can be directed to the corresponding author.

\section{AUTHOR CONTRIBUTIONS}

YL contributed to the conception of the study, contributed significantly to analysis and manuscript preparation, and performed the data analyses and wrote the manuscript. YL and EZ were responsible for the experiment. EZ helped perform the analysis with constructive discussions. WZ was responsible for the review and revision of the paper and supported the financial acquisition for the project leading to this publication. All authors contributed to the article and approved the submitted version.

GRO-alpha and leaves RANTES and MCP-2 intact. Blood. (2000) 96:2673-81. doi: 10.1182/blood.V96.8.2673

12. Zhu Y, Xian X, Wang Z, Bi Y, Chen Q, Han X, et al. Research progress on the relationship between atherosclerosis and inflammation. Biomolecules. (2018) 8:80. doi: 10.3390/biom 8030080

13. Sigala F, Kotsinas A, Savari P, Filis K, Markantonis S, Iliodromitis EK, et al. Oxidized LDL in human carotid plaques is related to symptomatic carotid disease and lesion instability. J Vasc Surg. (2010) 52:70413. doi: 10.1016/j.jvs.2010.03.047

14. Ro S, Park C, Young D, Sanders KM, Yan W. Tissue-dependent paired expression of miRNAs. Nucleic Acids Res. (2007) 35:594453. doi: 10.1093/nar/gkm641

15. Mallory AC, Vaucheret H. MicroRNAs: something important between the genes. Curr Opin Plant Biol. (2004) 7:120-5. doi: 10.1016/j.pbi.2004.01.006

16. Shirjang S, Mansoori B, Asghari S, Duijf PHG, Mohammadi A, Gjerstorff $M$, et al. MicroRNAs in cancer cell death pathways: Apoptosis and necroptosis. Free Radic Biol Med. (2019) 139:115. doi: 10.1016/j.freeradbiomed.2019.05.017

17. He Z, Wang $\mathrm{Y}$, He Q, Chen M. microRNA-491-5p protects against atherosclerosis by targeting matrix metallopeptidase-9. Open Med (Wars). (2020) 15:492-500. doi: 10.1515/med-2020-0047

18. Yuan M, Zhan Q, Duan X, Song B, Zeng S, Chen X, et al. functional polymorphism at miR-491-5p binding site in the 3'UTR of MMP-9 gene confers increased risk for atherosclerotic cerebral infarction in a Chinese population. Atherosclerosis. (2013) 226:447-52. doi: 10.1016/j.atherosclerosis.2012.11.026

19. Ye J, Wang C, Wang D, Yuan H. LncRBA GSA5, up-regulated by oxLDL, aggravates inflammatory response and MMP expression in THP-1 macrophages by acting like a sponge for miR-221. Exp Cell Res. (2018) 369:348-55. doi: 10.1016/j.yexcr.2018.05.039

20. Livak KJ, Schmittgen TD. Analysis of relative gene expression data using realtime quantitative PCR and the 2(-Delta Delta C(T)) Method. Methods. (2001) 25:402-8. doi: 10.1006/meth.2001.1262

21. Zhu Z, Li J, Zhang X. Astragaloside IV protects against oxidized lowdensity lipoprotein (ox-LDL)-induced endothelial cell injury by reducing oxidative stress and inflammation. Med Sci Monit. (2019) 25:213240. doi: 10.12659/MSM.912894 
22. Raggi P, Genest J, Giles JT, Rayner KJ, Dwivedi G, Beanlands RS, et al. Role of inflammation in the pathogenesis of atherosclerosis and therapeutic interventions. Atherosclerosis. (2018) 276:98108. doi: 10.1016/j.atherosclerosis.2018.07.014

23. Khan R, Spagnoli V, Tardif JC. L'Allier PL. Novel anti-inflammatory therapies for the treatment of atherosclerosis. Atherosclerosis. (2015) 240:497509. doi: 10.1016/j.atherosclerosis.2015.04.783

24. Hua Z, Ma K, Liu S, Yue Y, Cao H, Li Z. LncRNA ZEB1-AS1 facilitates ox-LDL-induced damage of HCtAEC cells and the oxidative stress and inflammatory events of THP-1 cells via miR-942/HMGB1 signaling. Life Sci. (2020) 247:117334. doi: 10.1016/j.lfs.2020.117334

25. Varani G. Twenty years of RNA: the discovery of microRNAs. RNA. (2015) 21:751-2. doi: 10.1261/rna.050237.115

26. Bu Q, Zhu Y, Chen QY Li H, Pan Y. A polymorphism in the 3'untranslated region of the matrix metallopeptidase 9 gene is associated with susceptibility to idiopathic calcium nephrolithiasis in the Chinese population. J Int Med Res. (2020) 48:300060520980211. doi: 10.1177/03000605209 80211

27. Sun R, Liu Z, Tong D, Yang Y, Guo B, Wang X, et al. miR-491-5p, mediated by Foxi1, functions as a tumor suppressor by targeting Wnt3a/ $\beta$-catenin signaling in the development of gastric cancer. Cell Death Dis. (2017) 8:e2714. doi: 10.1038/cddis.2017.134

28. Liu F, Zhang $\mathrm{H}$, Xie $\mathrm{F}$, Tao $\mathrm{D}$, Xiao $\mathrm{X}$, Huang $\mathrm{C}$, et al. Hsa_circ_0001361 promotes bladder cancer invasion and metastasis through miR-491-5p/MMP9 axis. Oncogene. (2020) 39:1696-709. doi: 10.1038/s41388-019-1092-z
29. Zhang H, Wang ZW, Wu HB Li Z, Li LC, Hu XP, et al. Transforming growth factor- $\beta 1$ induces matrix metalloproteinase- 9 expression in rat vascular smooth muscle cells via ROS-dependent ERK-NF-кB pathways. Mol Cell Biochem. (2013) 375:11-21. doi: 10.1007/s11010-012-1512-7

30. Walter L, Canup B, Pujada A, Bui TA, Arbasi B, Laroui H, et al. Matrix metalloproteinase 9 (MMP9) limits reactive oxygen species (ROS) accumulation and DNA damage in colitis-associated cancer. Cell Death Dis. (2020) 11:767. doi: 10.1038/s41419-020-02959-z

Conflict of Interest: The authors declare that the research was conducted in the absence of any commercial or financial relationships that could be construed as a potential conflict of interest.

Publisher's Note: All claims expressed in this article are solely those of the authors and do not necessarily represent those of their affiliated organizations, or those of the publisher, the editors and the reviewers. Any product that may be evaluated in this article, or claim that may be made by its manufacturer, is not guaranteed or endorsed by the publisher.

Copyright (c) 2021 Liao, Zhu and Zhou. This is an open-access article distributed under the terms of the Creative Commons Attribution License (CC BY). The use, distribution or reproduction in other forums is permitted, provided the original author(s) and the copyright owner(s) are credited and that the original publication in this journal is cited, in accordance with accepted academic practice. No use, distribution or reproduction is permitted which does not comply with these terms. 